\title{
Optimum pulse shapes for stimulated Raman adiabatic passage
}

\author{
G. S. Vasilev, ${ }^{1,2}$ A. Kuhn, ${ }^{1}$ and N. V. Vitanov ${ }^{2,3}$ \\ ${ }^{1}$ Department of Physics, University of Oxford, Parks Road, OX1 3PU Oxford, United Kingdom \\ ${ }^{2}$ Department of Physics, Sofia University, James Bourchier 5 blvd, 1164 Sofia, Bulgaria \\ ${ }^{3}$ Institute of Solid State Physics, Bulgarian Academy of Sciences, Tsarigradsko chaussée 72, 1784 Sofia, Bulgaria
}

(Dated: November 5, 2018)

\begin{abstract}
Stimulated Raman adiabatic passage (STIRAP), driven with pulses of optimum shape and delay has the potential of reaching fidelities high enough to make it suitable for fault-tolerant quantum information processing. The optimum pulse shapes are obtained upon reduction of STIRAP to effective two-state systems. We use the Dykhne-Davis-Pechukas (DDP) method to minimize nonadiabatic transitions and to maximize the fidelity of STIRAP. This results in a particular relation between the pulse shapes of the two fields driving the Raman process. The DDP-optimized version of STIRAP maintains its robustness against variations in the pulse intensities and durations, the single-photon detuning and possible losses from the intermediate state.

PACS numbers: 03.65.Ge, 32.80.Bx, 34.70.+e, 42.50.Vk
\end{abstract}

\section{INTRODUCTION}

Stimulated Raman adiabatic passage (STIRAP) is a well established and widely used technique for coherent population transfer in atoms and molecules [1]. STIRAP uses two delayed but partially overlapping laser pulses, pump and Stokes, which drive a three-state $\Lambda$ system $\psi_{1} \rightarrow \psi_{2} \rightarrow \psi_{3}$. The STIRAP technique transfers the population adiabatically from the initially populated state $\psi_{1}$ to the target state $\psi_{3}$. If the pulses are ordered counterintuitively, i.e. the Stokes pulse precedes the pump pulse, two-photon resonance is maintained, and adiabatic evolution is enforced, then complete population transfer from $\psi_{1}$ to $\psi_{3}$ occurs. Throughout this process, no population is placed in the (possibly lossy) intermediate state $\psi_{2}$. Various aspects of STIRAP have been subjects of intense research, both theoretically and experimentally [2].

Because STIRAP is an adiabatic technique it is insensitive to small to moderate variations in most of the experimental parameters, such as pulse amplitudes, widths, delay, and single-photon detuning. A particularly remarkable and very useful feature of STIRAP is its insensitivity to the properties of the intermediate state $\psi_{2}$. For instance, STIRAP has been demonstrated with high efficiency even for interaction durations exceeding the lifetime of $\psi_{2}$ by a factor of 100 [2]. For these reasons STIRAP is a very attractive technique for quantum information processing (QIP) [3, 4]. However, it is widely recognized that QIP requires very high fidelities, with the admissible error of gate operations being below $10^{-4}$ for a reliable quantum processor $[5,6]$. Such an extreme accuracy has not yet been demonstrated for STIRAP, as an accuracy of 90-95\% was sufficient for most traditional applications. When trying to increase the fidelity beyond this number one faces various obstacles related mainly to nonadiabatic transitions. Being an adiabatic technique, STIRAP reaches an efficiency of unity only in the adiabatic limit; however, the latter is approached only asymptotically as the pulse areas increase. For QIP, the pulse areas needed are so large that they may violate various restrictions of a real experiment.

In this paper we propose how to achieve an ultrahigh fidelity in STIRAP, and thus make it fully suitable for QIP by suitably shaped pulses. We utilize a recent idea of Guerin et al. [7] who applied the well-known DykhneDavis-Pechukas (DDP) method [8] to optimize adiabatic passage in a two-state system. In order to adapt this approach to STIRAP, we reduce the three-level Raman system to effective two-state systems in two limits: on exact resonance and for large single-photon detuning. The optimization, which minimizes the nonadiabatic transitions and maximizes the fidelity, leads to a particular relation between the pulse shapes of the driving pump and Stokes fields.

It should be noted that a fidelity of unity can also be achieved by a resonant $\pi$-pulse in a two-state transition. However, resonant techniques suffer from their sensitivity to parameter fluctuations. The optimized version of STIRAP presented here features both a very high fidelity and a robustness against variations in the intensities and the single-photon detuning.

This paper is organized as follows: In section II we review the DDP method and the optimization of two-state adiabatic passage. Then we extend this idea to STIRAP in section III and discuss examples in section IV. In section $\mathrm{V}$ we extend these ideas to fractional STIRAP (f-STIRAP), which creates a coherent superposition of $\psi_{1}$ and $\psi_{3}$. We summarize the results in the concluding section. 


\section{OPTIMIZATION OF ADIABATIC PASSAGE BETWEEN TWO STATES}

\section{A. Dykhne-Davis-Pechukas (DDP) approximation}

The probability amplitudes in a two-state system $\mathbf{a}(t)=\left[a_{1}(t), a_{2}(t)\right]^{T}$ satisfy the Schrödinger equation,

$$
\mathrm{i} \hbar \frac{\mathrm{d}}{\mathrm{d} t} \mathbf{a}(t)=\mathbf{H}(t) \mathbf{a}(t)
$$

where the Hamiltonian in the rotating-wave approximation (RWA) reads [9]

$$
\mathbf{H}(t)=\frac{1}{2} \hbar\left[\begin{array}{cc}
0 & \Omega(t) \\
\Omega(t) & 2 \Delta(t)
\end{array}\right] .
$$

The detuning $\Delta=\omega_{0}-\omega$ is the difference between the transition frequency $\omega_{0}$ and the carrier laser frequency $\omega$. The time-varying Rabi frequency $\Omega(t)=|d E(t)| / \hbar$ describes the laser-atom interaction, where $d$ is the electric dipole moment for the $\psi_{1} \leftrightarrow \psi_{2}$ transition and $E(t)$ is the laser electric field envelope.

A very accurate technique for deriving the transition probability in the near-adiabatic regime is the DykhneDavis-Pechukas (DDP) approximation [8]. The DDP formula gives the following expression for the probability for nonadiabatic transitions

$$
P \approx e^{-2 \operatorname{Im} D\left(t_{0}\right)}
$$

where

$$
D\left(t_{0}\right)=\int_{0}^{t_{0}} \varepsilon(t) d t
$$

is an integral over the splitting $\varepsilon(t)=\sqrt{\Omega(t)^{2}+\Delta(t)^{2}}$ of the eigenenergies of the Hamiltonian (2). The point $t_{0}$ (the transition point) is defined as the (complex) zero of the quasienergy splitting, $\varepsilon\left(t_{0}\right)=0$, which lies in the upper half of the complex $t$-plane (i.e., with $\operatorname{Im} t_{0}>0$ ). Equation (3) gives the correct asymptotic probability for nonadiabatic transitions provided: (i) the quasienergy splitting $\varepsilon(t)$ does not vanish for real $t$, including $\pm \infty$; (ii) $\varepsilon(t)$ is analytic and single-valued at least throughout a region of the complex $t$-plane that includes the region from the real axis to the transition point $t_{0}$; (iii) the transition point $t_{0}$ is well separated from the other quasienergy zero points (if any), and from possible singularities; (iv) there exists a level (or Stokes) line defined by $\operatorname{Im} D(t)=\operatorname{Im} D\left(t_{0}\right)$, which extends from $-\infty$ to $+\infty$ and passes through $t_{0}$.

For the case of multiple zero points in the upper $t$ plane, Eq. (3) can be generalized to include the contributions from all these $N$ zero points $t_{k}$ as

$$
P \approx\left|\sum_{k=1}^{N} \Gamma_{k} e^{\mathrm{i} D\left(t_{k}\right)}\right|^{2},
$$

where $\Gamma_{k}=4 \mathrm{i} \lim _{t \rightarrow t_{k}}\left(t-t_{k}\right) \dot{\vartheta}(t)$; usually $\Gamma_{k}=1$ or -1 . Here $\dot{\vartheta}(t)$ accounts for the nonadiabatic coupling between the adiabatic states, with $\vartheta(t)=\frac{1}{2} \tan ^{-1} \Omega(t) / \Delta(t)$.

\section{B. Optimization of two-state adiabatic passage}

Guérin et al. [7] have used the DDP method to optimize the adiabatic passage between two states in a very simple manner. Assuming that the probability for nonadiabatic losses is solely due to the transition points $t_{k}$, they have proposed to suppress these altogether by choosing the Rabi frequency $\Omega(t)$ and the detuning $\Delta(t)$ such that there are no transition points. This condition is obviously fulfilled if the quasienergy splitting is constant,

$$
\varepsilon(t)=\sqrt{\Omega(t)^{2}+\Delta(t)^{2}}=\text { const. }
$$

For example, this condition is fulfilled for a detuning and Rabi frequency defined as

$$
\begin{gathered}
\Delta(t)=\varepsilon_{0} \cos [\pi f(t)], \quad \Omega(t)=\varepsilon_{0} \sin [\pi f(t)], \\
0=f(-\infty) \leqq f(t) \leqq f(\infty)=1
\end{gathered}
$$

with $f(t)$ being an arbitrary monotonically increasing function with the above property. Condition (6) is not the only possible condition for adiabatic optimization, but it is the simplest one [7].

\section{OPTIMIZATION OF STIRAP}

\section{A. STIRAP}

The probability amplitudes of the three states in STIRAP $\mathbf{c}(t)=\left[c_{1}(t), c_{2}(t), c_{3}(t)\right]^{T}$ satisfy the Schrödinger equation,

$$
\mathrm{i} \frac{\mathrm{d}}{\mathrm{d} t} \mathbf{c}(t)=\mathbf{H}(t) \mathbf{c}(t)
$$

where the STIRAP Hamiltonian within the rotatingwave approximation (RWA) reads [9]

$$
\mathbf{H}(t)=\frac{1}{2}\left[\begin{array}{ccc}
0 & \Omega_{p}(t) & 0 \\
\Omega_{p}(t) & 2 \Delta & \Omega_{s}(t) \\
0 & \Omega_{s}(t) & 0
\end{array}\right] .
$$

The time-varying Rabi frequencies $\Omega_{p}(t)$ and $\Omega_{s}(t)$ describe the couplings between the intermediate state $\psi_{2}$ and, respectively, the initial state $\psi_{1}$ and the target final state $\psi_{3}$. STIRAP is easily explained with the so-called dark state $\varphi_{d}(t)$, which is a zero-eigenvalue eigenstate of $\mathbf{H}(t)$,

$$
\varphi_{d}(t)=\psi_{1} \cos \vartheta(t)-\psi_{3} \sin \vartheta(t)
$$

where the time-dependent mixing angle $\vartheta(t)$ is defined as

$$
\vartheta(t)=\tan ^{-1} \frac{\Omega_{p}(t)}{\Omega_{s}(t)} .
$$

The pulses in STIRAP are ordered counterintuitively, i.e., the Stokes pulse precedes the pump pulse,

$$
\lim _{t \rightarrow-\infty} \frac{\Omega_{p}(t)}{\Omega_{s}(t)}=0, \quad \lim _{t \rightarrow+\infty} \frac{\Omega_{s}(t)}{\Omega_{p}(t)}=0 .
$$


Then $0 \stackrel{-\infty \leftarrow t}{\longleftarrow} \vartheta(t) \stackrel{t \rightarrow \infty}{\longleftrightarrow} \pi / 2$, and therefore the dark state $\varphi_{d}(t)$ connects adiabatically states $\psi_{1}$ and $\psi_{3}$,

$$
\psi_{1} \stackrel{-\infty \leftarrow t}{\longleftarrow} \varphi_{d}(t) \stackrel{t \rightarrow \infty}{\longrightarrow}-\psi_{3} .
$$

Thus, if the evolution is adiabatic then the population passes from state $\psi_{1}$ to state $\psi_{3}$. Moreover, because the dark state does not contain a contribution from the (possibly lossy) intermediate state $\psi_{2}$, the properties of the latter are less important.

When the evolution is not completely adiabatic, the population transfer $\psi_{1} \rightarrow \psi_{3}$ might be incomplete. Moreover, the intermediate state receives some transient population, which may either be lost if state $\psi_{2}$ decays to other states, or lead to decoherence if it decays back into $\psi_{1}$ or $\psi_{3}$. For STIRAP to be a viable tool for quantum computing, nonadiabatic transitions have to be reduced below the fault-tolerance limit of $\lesssim 10^{-4}[5,6]$. We can estimate the required resources for STIRAP to reach such a fidelity as follows. The adiabatic condition for STIRAP (for $\Delta=0$ ) demands large pulse areas $A_{p, s}=\int_{-\infty}^{\infty} \Omega_{p, s}(t) \mathrm{d} t \gg 1$. This global condition is derived from the local adiabatic condition, which reads $\Omega(t) \gg|\dot{\vartheta}(t)|$. The probability for nonadiabatic transitions in the perturbative limit is $P_{\text {na }}(t) \sim \dot{\vartheta}^{2}(t) / \Omega^{2}(t)$; it measures the population that escapes to other adiabatic states and reduces the fidelity. The infidelity is therefore $1-P_{3} \sim 1 / A_{p, s}^{2}$. The fault-tolerance QIP limit therefore requires $A_{p, s} \gtrsim 100$. In fact, this very rough estimate neglects various details, such as the peculiarities of the nonadiabatic coupling $\dot{\vartheta}(t)$, and in reality the condition for the pulse areas is more restrictive.

In the following, we show that an optimized version of STIRAP can reach the fault-tolerance QIP limit by using much smaller pulse areas. In order to optimize STIRAP we use the same ideas as in the two-state adiabatic passage optimization by Guérin et al. [7]. To this end we make use of the reduction of STIRAP to effective twostate problems.

\section{B. Effective two-state systems}

\section{Single-photon resonance}

On single-photon resonance $(\Delta=0)$ the three-state system is reduced to an effective two-state system, with a detuning $\Omega_{s}(t)$ and a coupling $\Omega_{p}(t)[10,11]$,

$$
\mathrm{i} \frac{d}{d t}\left[\begin{array}{l}
b_{1}(t) \\
b_{2}(t)
\end{array}\right]=\frac{1}{2}\left[\begin{array}{cc}
\Omega_{s}(t) & \Omega_{p}(t) \\
\Omega_{p}(t) & -\Omega_{s}(t)
\end{array}\right]\left[\begin{array}{l}
b_{1}(t) \\
b_{2}(t)
\end{array}\right],
$$

where the probability amplitudes $b_{1,2}(t)$ are related to $c_{1,2,3}(t)$ as follows $[10,11]$

$$
\begin{aligned}
c_{1}(t)= & 2 \operatorname{Re}\left[b_{1}^{*}(t) b_{2}(t)\right] \sin \vartheta(t) \\
& +\left(\left|b_{1}(t)\right|^{2}-\left|b_{2}(t)\right|^{2}\right) \cos \vartheta(t), \\
c_{2}(t)= & 2 \operatorname{im}\left[b_{1}^{*}(t) b_{2}(t)\right], \\
c_{3}(t)= & 2 \operatorname{Re}\left[b_{1}^{*}(t) b_{2}(t)\right] \cos \vartheta(t) \\
& -\left(\left|b_{1}(t)\right|^{2}-\left|b_{2}(t)\right|^{2}\right) \sin \vartheta(t) .
\end{aligned}
$$

Because we have for STIRAP $\vartheta(-\infty)=0$ and $\vartheta(\infty)=$ $\pi / 2$, the initial condition $c_{1}(-\infty)=1$ demands the condition $\left|b_{1}(-\infty)\right|=1$ in the effective two-state system. The final-state population in STIRAP is

$$
\left|c_{3}(+\infty)\right|^{2}=\left[\left|b_{1}(+\infty)\right|^{2}-\left|b_{2}(+\infty)\right|^{2}\right]^{2} \text {. }
$$

Consequently, an optimized adiabatic evolution in the two-state system (14) implies optimized STIRAP. Hence applied to STIRAP, the two-state optimum condition (6) simply yields

$$
\Omega_{p}(t)^{2}+\Omega_{s}(t)^{2}=\Omega^{2}=\text { const. }
$$

In other words, the rms Rabi frequency should be constant. Again, as in the two-state optimization, this is not the only possible optimization condition but it is the simplest one.

\section{Far-off-resonance fields}

For large single-photon detuning $\Delta(t)$, the intermediate state can be eliminated adiabatically by setting $\dot{c}_{2}(t)=0$ in Eq. (8). We thus obtain [2]

$\mathrm{i} \frac{\mathrm{d}}{\mathrm{d} t}\left[\begin{array}{c}c_{1}(t) \\ c_{3}(t)\end{array}\right]=\frac{1}{2}\left[\begin{array}{cc}-\Delta_{\mathrm{eff}}(t) & \Omega_{\mathrm{eff}}(t) \\ \Omega_{\mathrm{eff}}(t) & \Delta_{\mathrm{eff}}(t)\end{array}\right]\left[\begin{array}{c}c_{1}(t) \\ c_{3}(t)\end{array}\right]$,

where the effective Rabi frequency $\Omega_{\mathrm{eff}}(t)$ and detuning $\Delta_{\text {eff }}(t)$ are defined as

$$
\begin{aligned}
\Omega_{\mathrm{eff}}(t) & =-\frac{\Omega_{p}(t) \Omega_{s}(t)}{2 \Delta(t)} \\
\Delta_{\mathrm{eff}}(t) & =\frac{\Omega_{p}(t)^{2}-\Omega_{s}(t)^{2}}{4 \Delta(t)}
\end{aligned}
$$

The two-state condition for optimal adiabatic passage now reads

$$
\Omega_{\mathrm{eff}}(t)^{2}+\Delta_{\mathrm{eff}}(t)^{2}=\left[\frac{\Omega_{p}(t)^{2}+\Omega_{s}(t)^{2}}{4 \Delta(t)}\right]^{2}=\text { const. }
$$

For constant $\Delta$ this condition is identical to the one we found on resonance, Eq. (17), that is it requires a constant rms Rabi frequency $\Omega$. We point out that, due to the identical conditions, on and off single-photon resonance, optimization is ensured over a very wide range of single-photon detunings. 


\section{OPTIMIZATION OF STIRAP: EXAMPLES}

\section{A. Pulse shapes}

Conditions (17) and (20) suggest the following parameterization of the pump and Stokes fields

$$
\begin{aligned}
& \Omega_{p}(t)=\Omega \sin \left[\frac{\pi}{2} f(t)\right], \\
& \Omega_{s}(t)=\Omega \cos \left[\frac{\pi}{2} f(t)\right],
\end{aligned}
$$

where $f(t)$ is an arbitrary monotonically increasing function, $0=f(-\infty) \leqq f(t) \leqq f(\infty)=1$. Viewed mathematically, $\Omega_{p}(t)$ and $\Omega_{s}(t)$ define an adiabatic path, for which the nonadiabatic correction given by the DDP formula vanishes, which leads to an optimal adiabatic following of the dark state.

The exact fulfillment of condition (17) requires a constant $\Omega$ and hence, infinite pulse areas. This unphysical condition can be overcome by using a "mask" function $F(t)$,

$$
\begin{aligned}
& \Omega_{p}(t)=\Omega_{0} F(t) \sin \left[\frac{\pi}{2} f(t)\right], \\
& \Omega_{s}(t)=\Omega_{0} F(t) \cos \left[\frac{\pi}{2} f(t)\right] .
\end{aligned}
$$

Then the rms Rabi frequency becomes time-dependent, $\Omega(t)=\Omega_{0} F(t)$. This replacement does not necessarily violate the optimization condition (17) because DDP transition points may still be absent, e.g. if the mask function has a suitable shape, such as Gaussian. Still, a pulse-shaped mask $F(t)$ violates the DDP conditions because the eigenenergy splitting $\varepsilon(t) \equiv \Omega_{0} F(t)$ becomes degenerate as $t \rightarrow \pm \infty$. The implication is that the probability for nonadiabatic transitions as a function of the pulse area is no longer expressed as a simple exponential, Eq. (3), but rather by a sum of an exponential and an oscillatory term with an amplitude that vanishes only polynomially with the pulse area $[11,12]$. The exponential term dominates for moderate pulse areas, whereas the oscillatory polynomial term dominates for large areas $[11,12]$. The border value of the area $A_{b}$, where the exponential decline breaks down into slowly damped oscillations, is proportional to the ratio $\mathrm{R}$ between the rms pulse area $A=\sqrt{A_{p}^{2}+A_{s}^{2}}$ and the overlap area $A_{o}$ (the area of overlap of the pump and Stokes pulses) $A_{b} \propto R=A / A_{o}$. The exponential decline of nonadiabatic transitions is favorable for high-fidelity STIRAP because it allows one to achieve high fidelity with moderate pulse areas. This is turn implies a large value of the breakdown area $A_{b}$, so that the (slowly damped) oscillations emerge only when the infidelity is very low. Hence high-fidelity STIRAP is facilitated by asymmetric pulses, with longer outer tails, so that the ratio $R$ (and hence the breakdown area $A_{b}$ ) is large. These observations are further illustrated with the figures below.

The above arguments suggest the following recipe for choosing $F(t)$ :

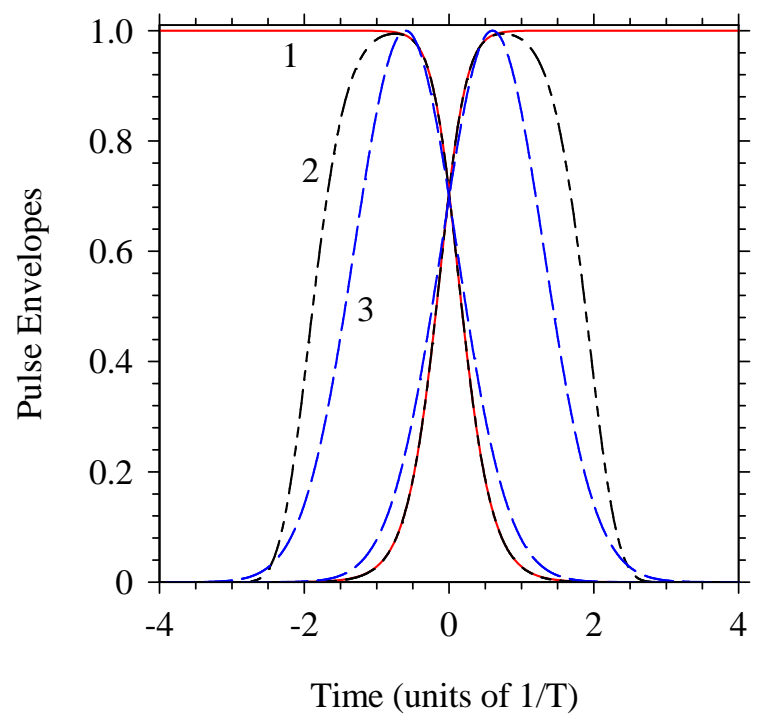

FIG. 1: Pulse shapes. 1: ideally optimized pulse shapes, which obey the optimization condition (17) for all times $t ; 2$ : pulse shapes with a hypergaussian mask (23), with $n=3$, $\lambda=4$ and $T_{0}=2 T ; 3$ : Gaussian pulses (27), with pulse delay $\tau=1.2 T$.

- $F(t)$ should be a flat function during the time of overlap of the pump and Stokes pulses, during which the population transition takes place;

- $F(t)$ should have a sufficiently large width so that the rms-to-overlap ratio $R$ is sufficiently large.

In the examples below, as a mask $F(t)$ we use the hypergaussian function,

$$
F(t)=\mathrm{e}^{-\left(t / T_{0}\right)^{2 n}}
$$

where $n=1$ corresponds to the Gaussian shape. For larger (positive integer) $n$ the condition $F(t) \simeq$ const is fulfilled increasingly better in the overlap region.

There is a some leeway in the choice of the function $f(t)$ as long as the adiabatic condition is fulfilled in the overlap region. We use

$$
f(t)=\frac{1}{1+\mathrm{e}^{-\lambda t / T}} .
$$

We point out that other choices of the function $f(t)$ are also possible. However, with the chosen method of optimization being based on the DDP approximation, which is valid only in the near-adiabatic regime, the function $f(t)$ has to fulfill the adiabaticity criterion

$$
|\dot{\vartheta}(t)| \ll|\varepsilon(t)|
$$

where $\dot{\vartheta}(t)$ is the nonadiabatic coupling. Using Eq. (25) we obtain the following condition for the function $f(t)$

$$
|\dot{f}(t)| \ll \Omega_{0}|F(t)|
$$




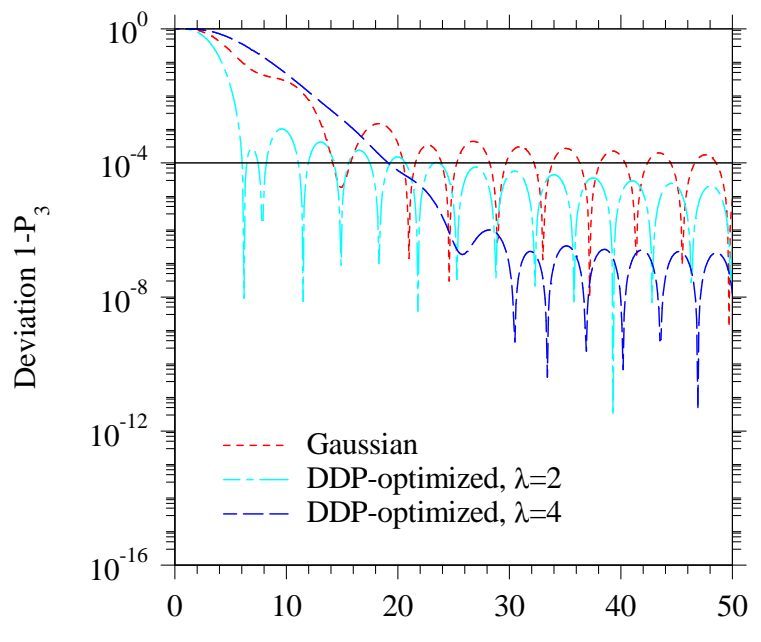

Peak Rabi Frequency (units 1/T)

FIG. 2: Deviation (infidelity) from complete population transfer vs the peak Rabi frequency for Gaussian (delay $\tau=1.2 T)$ and DDP-optimized pulses (23) $(n=3, \lambda=4$, $T_{0}=2 T$ and $n=1, \lambda=2, T_{0}=2 T$ ).

hence $f(t)$ should have a smooth time dependence in order to facilitate adiabaticity. Once in the adiabatic regime, however, the function $f(t)$ does not affect the DDP optimization because it does not appear in the condition (17).

Three different pulse shapes are shown in Fig. 1: pulse shapes that obey the optimization condition (17) for all times $t$, along with the more realistic example with a hypergaussian mask (23), that obey the optimization condition (17) only in the region of overlap of the pump and Stokes pulses, and Gaussian pulses.

$$
\Omega_{p}=\Omega_{0} \mathrm{e}^{-(t-\tau / 2) / T^{2}}, \quad \Omega_{s}=\Omega_{0} \mathrm{e}^{-(t+\tau / 2) / T^{2}},
$$

where $\tau$ is the pulse delay. We note that the pulse area $A$ for the Gaussian pulses is almost identical with the DDP optimized pulses. In the following we demonstrate that the optimally shaped pulses are superior to the Gaussian pulses, even with optimized delay for the latter, in achieving a very high fidelity.

\section{B. Examples of ultrahigh-fidelity STIRAP}

In Figure 2 we plot the STIRAP infidelity, i.e. the deviation $1-P_{3}$ from perfect transfer for the optimized pulses, described above, and compare these to the results for the traditional implementation of STIRAP with a pair of Gaussian pulses. The infidelity is shown as a function of the peak Rabi frequency. For Gaussian pulses, the pulse delay is chosen such that a nearly maximum fidelity is obtained. Despite the optimum delay, the Gaussian shapes do not allow us to reduce the infidelity below the limit $10^{-4}$ in the shown range of pulse areas (eventually, at very high pulse areas the infidelity drops below this

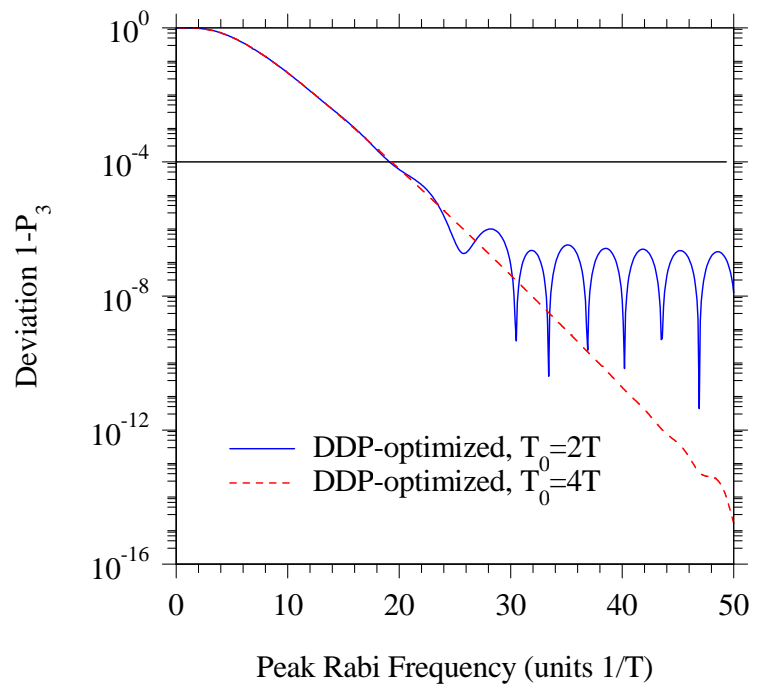

FIG. 3: Deviation (infidelity) from complete population transfer vs the peak Rabi frequency for DDP optimized pulses for different values of the width $T_{0}$ of the hypergaussian $(n=3)$ mask $F(t)$, Eq. (23).

limit). On the contrary, DDP-optimized pulse shapes (with $n=1, \lambda=2, T_{0}=2 T$ ) break this limit even for small pulse areas.

We note that the DDP pulses are not fully optimized due to the mask function $F(t)$ vanishing at large times. Therefore the respective fidelity curve starts to oscillate, which signals the occurence of nonadiabatic transitions. However, the magnitude of these nonadiabatic transitions can be controlled by the width of the mask function $F(t)$ : a larger width pushes these oscillations further down. Hence even for small pulse areas, the optimally shaped pulses are far superior to the Gaussian pulses, as shown in Fig. 2. This tendency is also visible in Fig. 3 where the infidelity is plotted as a function of the pulse area for two different widths $T_{0}$ of the mask function $F(t)$, Eq. (23). By increasing the mask width $T_{0}$, the validity range of the adiabatic optimization condition (17) widens, and the optimized pulses approach the ideal DDP-optimized pulse shapes in Fig. 1.

In Fig. 4 we compare the line profile as a function of the common detuning $\Delta$ from the intermediate level (see Eq. (9)) for the optimized and Gaussian pulse shapes. The time delay for the Gaussian pulses is numerically chosen for nearly maximum fidelity. It is known that a singlephoton detuning does not affect the dark state (as long as two-photon resonance is maintained) [2]. Nonetheless, adiabaticity deteriorates and the transfer efficiency decreases with increasing single-photon detuning. The robustness of the high fidelity STIRAP (i.e. STIRAP where the infidelity is below the limit $10^{-4}$ ) against the single-photon detuning is much more pronounced for the optimized pulses. This feature is readily explained by the fact that the same pulse shapes optimize STIRAP both for $\Delta=0$ and for large $\Delta$, as discussed above. 


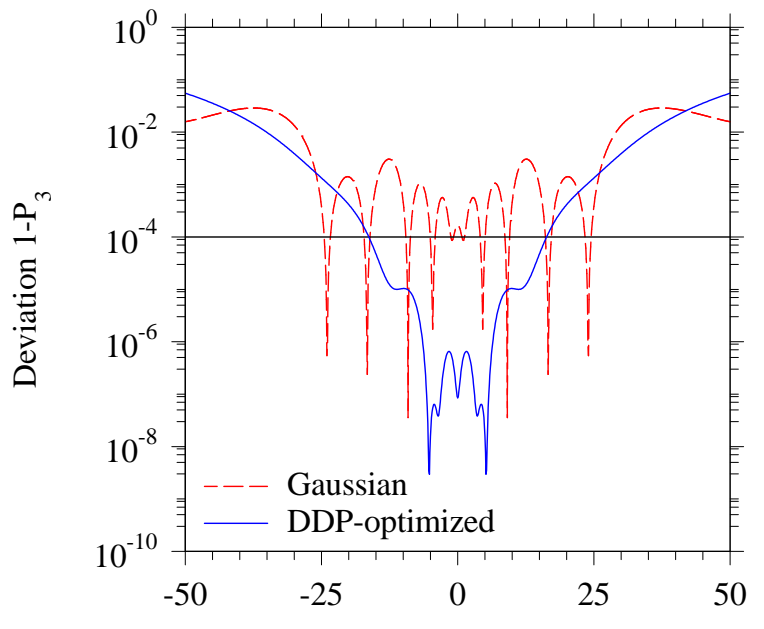

Single-Photon Detuning (units 1/T)

FIG. 4: Line profile as a function of the common detuning $\Delta$ from the intermediate level for DDP-optimized ( $n=3, \lambda=4$, $T_{0}=2 T$ ) and Gaussian pulses (delay $\tau=1.2 T$ ) for a peak Rabi frequency $\Omega=20$.

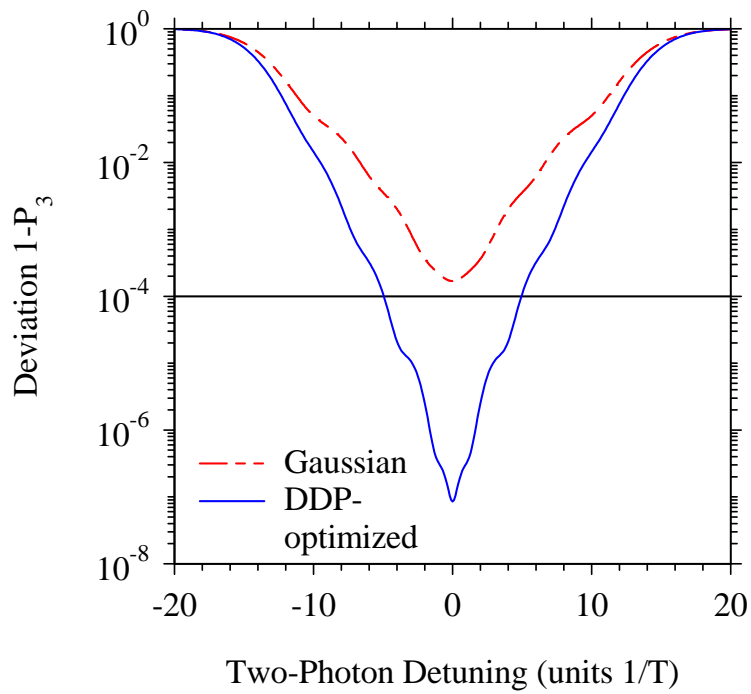

FIG. 5: Raman line profile for the DDP-optimized ( $n=3$, $\lambda=4 T_{0}=2 T$ ) and Gaussian pulses (delay $\tau=1.2$ ) for a peak Rabi frequency $\Omega=20$.

It is known that the transfer efficiency for STIRAP is much more sensitive to a detuning from Raman resonance than to the single-photon detuning [2]. Figure 5 shows the Raman line profile for DDP-optimized and Gaussian pulses. As before, the time delay for the Gaussian pulses is numerically chosen such that a nearly maximum transition probability $P_{3}$ is obtained. The optimized pulse shapes are far superior to the Gaussian pulses and allow one to maintain a high fidelity over a wide range of two-photon detunings.

\section{Relative error due to the RWA approximation}

The rotating wave approximation (RWA) is widely used whenever laser-induced excitations with optical frequencies $\omega$ much larger than the Rabi frequency $\Omega$ are considered. Typical optical (carrier) frequencies are $\omega \sim 10^{16}\left[s^{-1}\right]$, while the typical Rabi frequencies are within the range $\Omega \sim 10^{8}-10^{9}\left[s^{-1}\right]$ [9]. For two-level systems, perturbative inclusion of the counter-rotating terms results in the Bloch-Siegert shift of the eigenenergies by $\sim \Omega^{2} / \omega[17]$. Within a typical pulse duration $T \sim 10^{-6}-10^{-8}[s]$, this accumulates maximum deviation from the calculated probabilities of

$$
\triangle P_{e} \sim\left(T \frac{\Omega^{2}}{\omega}\right)^{2} \simeq 10^{-8}-10^{-16}
$$

It should be also noted that the results in RWA are exact in case of circular polarization [9].

\section{OPTIMIZATION OF HADAMARD GATES}

Fractional STIRAP (f-STIRAP) is a variation of STIRAP, which creates an arbitrary preselected coherent superposition of states $\psi_{1}$ and $\psi_{3}$,

$$
\Psi=\psi_{1} \cos \alpha-\psi_{3} \sin \alpha .
$$

As in STIRAP, the Stokes pulse precedes the pump pulse, but unlike STIRAP, where the Stokes pulse vanishes first, in f-STIRAP the two pulses vanish simultaneously, while maintaining a constant ratio of their amplitudes [13],

$$
0 \stackrel{-\infty \leftarrow t}{\longleftarrow} \frac{\Omega_{p}(t)}{\Omega_{s}(t)} \stackrel{t \rightarrow \infty}{\longrightarrow} \tan \alpha .
$$

A convenient realization of f-STIRAP reads [14]

$$
\begin{aligned}
& \Omega_{p}(t)=\Omega_{0} \mathrm{e}^{-(t-\tau / 2)^{2} / T^{2}} \sin \alpha, \\
& \Omega_{s}(t)=\Omega_{0}\left\{\mathrm{e}^{-(t+\tau / 2)^{2} / T^{2}}+\mathrm{e}^{-(t-\tau / 2)^{2} / T^{2}} \cos \alpha(31 \mathrm{~b})\right.
\end{aligned}
$$

The DDP-optimized pulses, in analogy with the full STIRAP, read

$$
\begin{aligned}
& \Omega_{p}(t)=\Omega_{0} F(t) \sin [\alpha f(t)], \\
& \Omega_{s}(t)=\Omega_{0} F(t) \cos [\alpha f(t)],
\end{aligned}
$$

where $f(t)$ is again an arbitrary function that satisfies condition (7b). It is easy to verify that these pulse shapes satisfy the f-STIRAP condition (30). For half-STIRAP, when an equal coherent superposition of states $\psi_{1}$ and $\psi_{3}$ is created, we should have $\alpha=\pi / 4$. This superposition corresponds to the Hadamard gate, which is one of the fundamental gates in quantum information processing.

Figure 6 compares the transfer efficiency of f-STIRAP for DDP-optimized pulses and for pulses given by Eqs. 


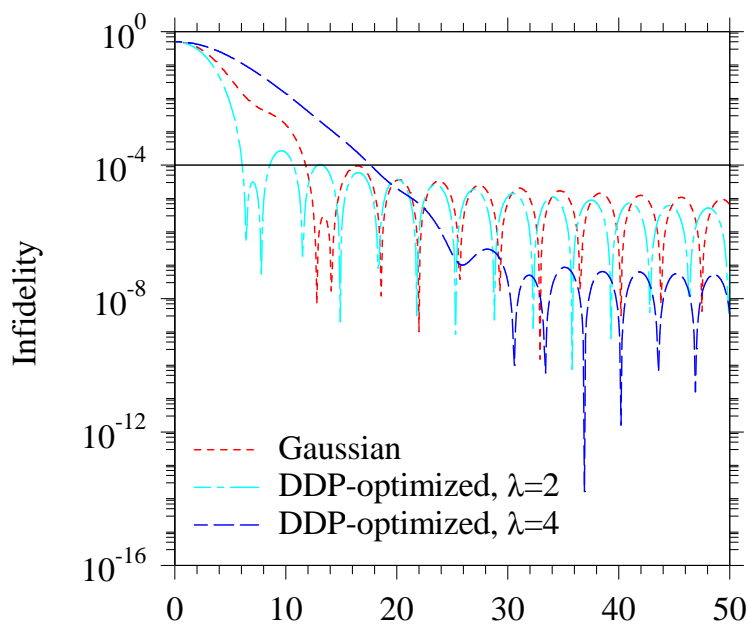

Peak Rabi Frequency (units 1/T)

FIG. 6: Transfer efficiency for fractional STIRAP vs the peak Rabi frequency for DDP-optimized pulses (with $n=3, \lambda=4$, $T_{0}=2 T$ and $n=1, \lambda=2, T_{0}=2 T$ ) and Gaussian pulses (31) (with $\tau=1.4 T$ ).

(31). The pulse delay for the pulses (31) is chosen numerically for nearly maximum fidelity. In Fig. 6 the infidelity is defined as

$$
1-\left|<\Psi_{\text {final }}\right| \Psi_{\text {desired }}>\left.\right|^{2},
$$

where $\Psi_{\text {desired }}$ is given by Eq. (29) at $\alpha=\pi / 4$ and $\Psi_{\text {final }}$ is numerically calculated for both DDP-optimized and Gaussian pulses. It is important to note that despite the delay optimization, the pulses (31) do not allow reduction of the infidelity below a certain limit. Due to the mask function $F(t)$, the DDP-optimized pulses led to an oscillatory behaviour of the infidelity. However, the oscillatory regime and hence the fidelity depend on the width $T_{0}$ and can be controlled. In particular in the regime of small pulse areas, the DDP-optimized version of f-STIRAP (with $n=1, \lambda=2, T_{0}=2 T$ ) is far superior to the traditional Gaussian pulses as shown in Fig. 6 .

\section{CONCLUSIONS}

We have proposed an optimization of the fidelity of the STIRAP technique, which uses the DDP approach to minimize nonadiabatic losses. The rationale for this is the reduction of STIRAP from three to two states either: on exact single-photon resonance or for large singlephoton detuning. Interestingly, the optimized pulse shapes are the same in both regimes, which makes DDPoptimized STIRAP very robust against variations in the detuning. We have demonstrated with numerical simulations that the fidelity of this optimized STIRAP can reach very high values, with an error well below the faulttolerant QIP limit of $10^{-4}$, which is very difficult to reach by optimizing the pulse delay with the usual Gaussian pulse shapes (or other symmetric pulse shapes, such as hyperbolic-secant). The proposed optimization is of potential importance for QIP because it supplements the robustness of STIRAP against parameter variations with an ultrahigh fidelity of gate operations. We furthermore emphasize that a similar optimization method could also have a significant impact on the vacuum-stimulated Raman scattering in cavity QED [16].

\section{Acknowledgments}

This work has been supported by the projects EMALI, FASTQUAST and SCALA of the European Union, the EPSRC grant(EP/E023568/1), the research unit 635 of the German research foundation and the Bulgarian NSF Grants Nos. 205/06, 301/07, and D002-90/08.
[1] U. Gaubatz, P. Rudecki, S. Schiemann, K. Bergmann, J. Chem. Phys. 92, 5363 (1990); S. Schiemann, A. Kuhn, S. Steuerwald, K. Bergmann, Phys. Rev. Lett. 71, 3637 (1993).

[2] N. V. Vitanov, M. Fleischhauer, B. W. Shore and K. Bergmann, Adv. At. Mol. Opt. Phys. 46, 55 (2001); N. V. Vitanov, T. Halfmann, B. W. Shore and K. Bergmann, Ann. Rev. Phys. Chem. 52, 763 (2001); J. Klein, F. Beil, and T. Halfmann, Phys. Rev. A 78, 033416 (2008).

[3] M. Hennrich, T. Legero, A. Kuhn and G. Rempe, Phys. Rev. Lett. 85, 4872 (2000); A. Kuhn, M. Hennrich, and G. Rempe, Phys. Rev. Lett. 89, 067901 (2002).

[4] Z. Kis and F. Renzoni, Phys. Rev. A 65, 032318 (2002); X. Lacour, S. Guérin, N. V. Vitanov, L. P. Yatsenko and H. R. Jauslin, Opt. Commun. 264, 362 (2006); C. Wunderlich, T. Hannemann, T. Körber, H. Häffner, C. Roos, W. Hänsel, R. Blatt and F. Schmidt- Kaler, J. Mod. Opt.
54, 1541 (2007).

[5] M. A. Nielsen and I. L. Chuang, Quantum Computation and Quantum Information (Cambridge University Press, 1990).

[6] P. W. Shor, 37th Symposium on Foundations of Computing 56-65 (IEEE Computer Society Press, Washington DC, 1996); A. Steane, Rep. Prog. Phys. 61, 117 (1998); E. Knill, Nature 434, 39 (2005); J. Benhelm, G. Kirchmair, C. F. Roos and R. Blatt, Nature Phys. 4, 463 (2008).

[7] S. Guérin, S. Thomas, and H. R. Jauslin, Phys. Rev. A 65, 023409 (2002); X. Lacour, S. Guérin and H. R. Jauslin, Phys. Rev. A 78, 033417 (2008).

[8] J. P. Davis and P. Pechukas, J. Chem. Phys. 64, 3129 (1976); A. M. Dykhne, Sov. Phys. JETP 11, 411 (1960).

[9] B. W. Shore, The Theory of Coherent Atomic Excitation (Wiley, New York, 1990). 
[10] T. A. Laine and S. Stenholm, Phys. Rev. A 53, 2501 (1996).

[11] N. V. Vitanov and S. Stenholm, Opt. Commun. 127, 215 (1996).

[12] K. Drese and M. Holthaus, Eur. Phys. J. D 3, 73 (1998).

[13] P. Marte, P. Zoller and J. L. Hall, Phys. Rev. A 44, R4118 (1991).

[14] N. V. Vitanov, K.-A. Suominen and B. W. Shore, J. Phys. B: At. Mol. Opt. Phys. 32, 4535 (1999).

[15] J. Volz, M. Weber, D. Schlenk, W. Rosenfeld, J. Vrana,
K. Saucke, C. Kurtsiefer and H. Weinfurter, Phys. Rev. Lett. 96, 030404 (2006).

[16] T. Wilk, S. C. Webster, H. P. Specht, G. Rempe, and A. Kuhn, Phys. Rev. Lett. 98, 063601 (2007); A. Kuhn, M. Hennrich, and G. Rempe, Phys. Rev. Lett. 89, 067901 (2002).

[17] M. P. Silverman and F. M. Pipkin, J. Phys. B:Atom. Molec. Phys. 5, 1845 (1972). 